\title{
(c) 19 \\ Relación entre la Planificación Estratégica y Gestión del Conocimiento
}

\author{
Relationship between Strategic Planning and Knowledge Management \\ Relação entre Planejamento Estratégico e Gestão do Conhecimento
}

Fiorella Gomez Zuñiga

\section{RESUMEN}

En la actualidad, las instituciones muestran gran preocupación por la planificación de sus objetivos y actividades, asimismo requieren de conocimientos necesarios que permita cubrir las expectativos de sus usuarios o clientes. De ahí la necesidad de que conozcan estrategias de planificación y gestionen el conocimiento a fin de maximizar sus ganancias. por ello, este artículo tiene como finalidad analizar la relación entre la planificación estratégica y la gestión del conocimiento, específicamente dentro de la academia. Para ello, se realizó un estudio descriptivo correlacional, se usaron dos cuestionarios para el desarrollo de las dimensiones. Los resultados señalan que, definitivamente, se establece una correlación entre ambas variables en los profesores de la muestra elegida. También, se evidencia que hay correlación entre estas dimensiones de la planificación estratégica y las dimensiones de la gestión del conocimiento. Además, se encuentra del mismo modo, que los colaboradores de las sedes de LimaMetropolitana los cuales se ubican en cinco distritos, sobre todo en Cercado de Lima.

Palabras clave: Planificación estratégica; Calidad; Gestión del conocimiento; Iniciativa organizacional; Satisfacción laboral
ABSTRACT

RESUMO
Nowadays, institutions show great concern for the planning of their objectives and activities, and they also require the necessary knowledge to meet the expectations of their users or clients. Hence the need for them to know planning strategies and manage knowledge in order to maximize their profits. Therefore, the purpose of this article is to analyze the relationship between strategic planning and knowledge management, specifically within the academy. For this purpose, a descriptive correlational study was carried out, using two questionnaires for the development of the dimensions. The results indicate that there is definitely a correlation between both variables in the teachers of the chosen sample. Also, it is evident that there is correlation between these dimensions of strategic planning and the dimensions of knowledge management. In addition, it is also found that the collaborators of the Lima-Metropolitan headquarters are located in five districts, mainly in Cercado de Lima.

Key words: Strategic planning; Quality; Knowledge management; Organizational initiative; Job satisfaction
Atualmente, as instituições demonstram grande preocupação com o planejamento de seus objetivos e atividades, e também exigem o conhecimento necessário para atender às expectativas de seus usuários ou clientes. Daí a necessidade de conhecer estratégias de planejamento e gerenciar o conhecimento a fim de maximizar seus lucros. Portanto, este artigo visa analisar a relação entre planejamento estratégico e gerenciamento do conhecimento, especificamente dentro da academia. Para este fim, foi realizado um estudo descritivo correlacional, utilizando dois questionários para o desenvolvimento das dimensões. Os resultados mostram que existe definitivamente uma correlação entre as duas variáveis nos professores da amostra escolhida. É também evidente que existe uma correlação entre estas dimensões do planejamento estratégico e as dimensões da gestão do conhecimento. Além disso, constata-se também que os colaboradores da sede de LimaMetropolitan estão localizados em cinco distritos, principalmente no Cercado de Lima.

Palavras-chave: Planejamento estratégico; Qualidade; Gestão do conhecimento; Iniciativa organizacional; Satisfação no trabalho 


\section{INTRODUCCIÓN}

Desde el acontecimiento de la gran revolución industrial el individuo era considerado como un agente reemplazable en el proceso productivo. Esto es, tenía el mismo valor y trato que una máquina o parte de esta, por lo cual se podría prescindir o reemplazar de acuerdo al criterio de los empleadores o del propietario. No obstante, los continuos cambios que comienzan a producirse a partir de la segunda mitad del siglo XX, en especial a nivel de las empresas o instituciones, empiezan a modificar la concepción o idea que se tiene sobre el trabajador o colaborador que progresivamente se fue cambiando la gestión de los colaboradores, por consiguiente, es indispensable en una organización. Resulta indispensable realizar una constante verificación a la calidad educativa, así como, a las políticas educativas recientes con la finalidad de medir el nivel de asimilación del conocimiento que adquieren los educados como parte del proceso que realizan con la ayuda del facilitador. Por lo tanto, es necesario establecer una relación entre estos conceptos estudiados (Díaz, 2015).

En este contexto novedoso a nivel empresarial u organizacional, se concibieron nuevas y teorías en cuanto a la gestión, que aplicadas a las organizaciones ofrecerán resultados bastanteóptimos para el mundo empresarial, por lo cual se empieza a extender a todas partes del mundo. Una empresa encaminada hacia la búsqueda de la excelencia o calidad se transforma en promotora de una cultura que se muestra a través de los comportamientos, conductas, labores y procesos para facilitar valor a través del cumplimiento de las necesidades y expectativas de los involucrados y otros agentes interesados. (Ramírez-Betancourt et al., 2020). Aquellos hacen referencia, esencialmente, al desarrollo, difusión del conocimiento y su adecuada gestión, de modo que, todos manejen la información y puedan tener un mejor resultado que se centra sobre todo en reunir, analizar, almacenar y compartir el conocimiento e información existente en la empresa, de tal manera que, pueda garantizarse los resultados esperados a partir de las decisiones que se tomen. Para ello se debe considerar a los recursos humanos de área administrativa en quienes se depositan la confianza de hacer un diagnóstico que permite tomar los mejores planes de emprendimiento para dar solución a las dificultades o retos que se tengan que asumir.

De modo que, en la actualidad el conocimiento se considera un activo intangible de primordial importancia para las organizaciones o instituciones, por lo cual su tratamiento merece una consideración especial. Dentro del campo educativo son ellos quienes a través de su labor mediadora incluso incentivan a su alumnado a conocer más de lo que ya se le proporciona. Es decir, los colaboradores desempeñan una labor crucial. Los empleadores deben cambiar obligatoriamente la forma de relacionarse con cada uno de los agentes que intervengan en la entidad. Según Aranda, y Salgado (2005) afirma que la educación pretende, por tanto, formar individuos socialmente adaptados, es decir, capaces de ser responsables de ciertas funciones sociales.

Por otro lado, dichas maniobras que conllevan al éxito de la institución demandan de mucho compromiso y participación activa de los colaboradores, con el fin de generar el conocimiento pertinente para cumplir con las metas expresadas a principio de cada proceso. Si estos tendrían manejo de dicha información se podría enfrentar de distinta perspectiva los problemas. Bajo estas situaciones 
es que surge la planificación estratégica como un elemento esencial al interior de las empresas $\mathrm{u}$ organizaciones en la medida de que está vinculada a las acciones que se ejecutarán para alcanzar las metas. Núñez y Rodríguez (2015) refieren que las organizaciones se están enfrentando a múltiples cambios en su entorno, el cual se caracteriza por ser sumamente complejo y competitivo, entorno del cual las IES no pueden abstraerse y mucho menos aislarse. De modo que, en todos los niveles de educación es necesario un plan estratégico que rija toso su accionar.

Dicho plan puesto en acción en la organización tiene como uno de sus cimientos para el éxito del mismo, mantenerse demasiado vinculado a la gerencia o área de gestión de tal manera que pueda ser capaz de modificar la visión y misión en los resultados deseados por la organización. De este modo, se optimizaría el tiempo operativo, igualmente el número de conflictos, dificultades o desafíos y lograría la participación activa de los colaboradores en la perspectiva de lograr el futuro planteado. La entidad u organización debe fidelizar a sus empleados; para que, hagan suya esa visión y misión.

Esta primera variable planteada en la investigación se ha transfigurado en una decisión destinada a garantizar el desarrollo humano, la presente investigación tiene como propósito promoverlo, en el afán de cambiar y favorecer el trabajo en equipo, que, sin lugar a dudas, debe colaborar a que las entidades educativas de los distintos niveles operen correctamente y así cumplan sus metas, camine hacia su visión y sea sostenible a través de los años. Esta planificación es determinante porque garantiza alcanzar las expectativas institucionales.
Para Ojeda (2013) la planificación estratégica en el sector educativo tuvo un desfase de 10 años a diferencia de otros sectores. Algunas instituciones de hoy no siguen un plan estratégico que les sirva de guía u oriente, para que, tomen decisiones acertadas y más aún en el plano educativo falta tener un plan y que se cumpla. Por ello, es indispensable contar uno, ya que, puede directamente relacionarse con la gestión del conocimiento. Además, considero que no se cuenta con el camino que deben tomar, para que, las decisiones regulen el desempeño de las entidades, de modo que, se desencadene en una óptima gestión del conocimiento. Por lo mencionado es necesario esta investigación para que vean la importancia que tiene contar un plan estratégico en base al análisis circunstancial y objetivo de la entidad.

El objetivo del estudio es analizar la relación entre la planificación estratégica y la gestión del conocimiento, específicamente dentro de la academia, ya que, se busca esclarecer que existe una relación directamente proporcional entre una óptima planificación estratégica que garantizaría que haya una acertada gestión del conocimiento. En base a los antecedentes revisados y las evidencias halladas en el proceso se demuestra la importancia de esta relación para el logro de las metas institucionales.

\section{MÉTODO}

Se realizó un estudio en el distrito de Cercado de Lima en Perú. El diseño de la investigación fue de tipo descriptivo bajo el análisis correlacional. Asimismo, para la recolección de la información fueron empleados dos cuestionarios, el primero de ello, estuvo constituido por treinta ítems bajo la estructura de escala de Likert, en la cual se 
obtuvo valoraciones de la variable Planificación Estratégica cuyo tiempo límite fue de 15 minutos, desarrollando las siguientes dimensiones FODA, PEI, Visión, Misión y Proceso de planeación; el segundo cuestionario constó de cuarenta ítems para Gestión del Conocimiento, a través de la apreciación de esta variable. El tiempo estimado fue de 25 minutos. Por otro lado, la muestra la conformaron 101 docentes de la institución educativa particular "Saco Oliveros". Se trabajó y fueron considerado la totalidad de los maestros que imparten sus clases en el nivel preuniversitario en la institución, es decir, Academias Saco Oliveros.

En este estudio se consideraron datos que fueron trabajados para garantizar la validez de los resultados. Para ello se accedió adecuadamente a la muestra de docentes y aplicó los instrumentos garantizando el anonimato de los agentes intervinientes. Por otro lado, los instrumentos empleados fueron dos cuestionarios tal como se menciona anteriormente. En el cual el cuestionario de planificación estratégica es confiable en la medida que el coeficiente Alfa de Cronbach obtuvo un valor total de 0,89 el cual se califica como alto. En cuanto a la validez, el resultado del análisis estadístico indica que la prueba estuvo conformada por un solo factor que en general explica el 70,48\% de la varianza total. Por otra parte, se obtuvo que la medida de adecuación del muestreo de KaiserMeyer-Olkin alcanza un valor de 0,76 que puede considerarse como adecuado, mientras que el test de esfericidad de Bartlett presenta un valor que es significativo.
En lo que respecta al cuestionario de gestión del conocimiento, todos los ítems forman parte de dicho cuestionario, y corresponden a cada una de las sub escalas asignadas por su autora. El coeficiente Alfa de Cronbach obtuvo un valor total de 0,97 el cual se puede calificar como alto, lo cual indica que la escala es confiable.

Los resultados del análisis estadístico de la prueba indican que está conformada por un solo factor, que explica el $83,14 \%$ de la varianza total. Por otra parte, tenemos que la medida de adecuación del muestreo de Kaiser-Meyer-Olkin alcanza un valor de 0,95 que puede considerarse como adecuado, mientras que el test de esfericidad de Bartlett presenta un valor que es significativo.

\section{RESULTADOS Y DISCUSIÓN}

A partir de la revisión y análisis de los temas tratados se evidencia que es determinante la planificación estratégica en la práctica docente y para tener en cuanta ese grado de significancia se observaron los resultados del test aplicados entre las dimensiones de la Planificación Estratégica y las dimensiones de la Gestión del conocimiento como se destaca en la Tabla 1, a un grupo de docentes de diferentes áreas del conocimiento quienes desde su experiencia dedujeron que su labor logra mejores resultados con un adecuada gestión del conocimiento que se respalda por la adecuada planificación estratégica de sus actividades, asimismo a través de las pruebas ejecutadas. 
Tabla 1. Planificación estratégica y las dimensiones de la gestión del conocimiento.

\begin{tabular}{lcccccccc}
\hline Variables & CreaC & ComC & ApliC & Creat & Innov & Conf & InfI & InfE \\
\hline Proceso de planificación & $0,60^{* *}$ & $0,61^{* *}$ & $0,55^{* *}$ & $0,55^{* *}$ & $0,55^{* *}$ & $0,54^{* *}$ & $0,51^{* *}$ & $0,59^{* *}$ \\
Visión & $0,67^{* *}$ & $0,69^{* *}$ & $0,55^{* *}$ & $0,63^{* *}$ & $0,64^{* *}$ & $0,64^{* *}$ & $0,67^{* *}$ & $0,68^{* *}$ \\
Misión & $0,54^{* *}$ & $0,60^{* *}$ & $0,39^{* *}$ & $0,48^{* *}$ & $0,55^{* *}$ & $0,52^{* *}$ & $0,56^{* *}$ & $0,57^{* *}$ \\
PEI & $0,54^{* *}$ & $0,60^{* *}$ & $0,49^{* *}$ & $0,51^{* *}$ & $0,54^{* *}$ & $0,48^{* *}$ & $0,52^{* *}$ & $0,57^{* *}$ \\
FODA & $0,55^{* *}$ & $0,64^{* *}$ & $0,49^{* *}$ & $0,49^{* *}$ & $0,56^{* *}$ & $0,51^{* *}$ & $0,60^{* *}$ & $0,59^{* *}$ \\
\hline
\end{tabular}

Como se puede observar en esta tabla a través de los resultados obtenidos. Se pudo concluir que si existe una relación significativa entre los conceptos estudiados.

Por otro lado, a través del análisis de los resultados se determinó que realmente si hay una estrecha relación entre la Planificación Estratégica y la Gestión del conocimiento y que no necesariamente se da a nivel de la educación básica regular, sino también a nivel superior donde suelen ser más determinantes las decisiones porque pueden conllevar a desastres sino hay monitoreo del todo el proceso que se realice. Al aplicar un plan estratégico se logró una mejor gestión del conocimiento. Por ello, se concluye que la Hipótesis planteada en un inicio fue cierta y respaldada con las evidencias de las respuestas anónimas recibidas.

Los hallazgos obtenidos señalan que existen correlaciones entre los conceptos en estudio, así que, las inferencias planteadas se vuelven a ratificar. Estos resultados confirman las investigaciones realizadas de otros investigadores quienes se centraron en la gestión del conocimiento en distintos contextos.

\section{Discusión}

Por esta parte, se tiene la certeza de que se emplearon adecuados instrumentos que respondían al fenómeno observado. En la dinámica de la emergente llamada sociedad del conocimiento, la economía del conocimiento adquiere un papel determinante en los sistemas sociales y culturales de todas las naciones. Los sistemas educativos que, a partir del siglo pasado, se convierten en una de las piezas angulares del desarrollo económico y social de los estados nacionales, hoy se encuentran inmersos en las transformaciones de la sociedad del conocimiento y de la economía del conocimiento (Minakata, 2020).

En el complejo escenario en el que desarrollan su trabajo las organizaciones modernas, incrementa la importancia del empleo de algún sistema de planificación estratégica que sirva de guía para la realización efectiva de su misión, así como para la conformación de una idea más o menos precisa sobre cómo deberán proyectarse en el futuro cercano, cuestión esta última a la que los especialistas suelen denominar visión (Concepción, 2007). Demuestra una vez más que la acción y las medidas que se tomen oportunamente suelen ser determinante para un gran cambio en las entidades. Asimismo, Dunorme (2012), sostiene que "la planificación estratégica, es una herramienta de gestión que permite apoyar la toma de decisiones de las organizaciones en torno al quehacer actual y al camino que deben recorrer en el futuro para adecuarse a los cambios y a las demandas que les impone el entorno y lograr la mayor eficiencia, eficacia, calidad en los bienes y servicios que se proveen", (p. 22). 
Se debe considerar, siguiendo a Armijo (2011) que "la planificación estratégica es un proceso continuo que requiere constante retroalimentación acerca de cómo están funcionando las estrategias". Finalmente, el test refleja que presenta adecuada aproximación a la curva normal, por lo que según Siegel y Castellan (1995) señaló "Es factible utilizar para el análisis de los datos, estadísticas no paramétricas". Las evidencias halladas demostraron que al plantear la hipótesis acerca de la relación entre ambos conceptos estudiados en los profesores de la institución analizada. Con los hallazgos denotaron que la primera dimensión, así como, las demás dimensiones del primer concepto mencionado establecen una relación de manera significativa con cada una de las dimensiones de la segunda variable.

\section{CONCLUSIONES}

A partir del análisis de los documentos y las lecturas revisadas y los resultados hallados en todo el proceso se concluye que si existe una relación significativa entre la planificación estratégica y la gestión el conocimiento. Por lo tanto, es importante que se aplique a nivel institucional y personal que como se vio, contribuye al logro de los objetivos Smart que se proponga como persona jurídica o persona natural. Asimismo, la planificación estratégica es un gran recurso, para que, se cumpla al 100\% cada una de las actividades, de modo que, se garantice que la institución no actúa de modo improvisado, sino que todo es resultado de un trabajo colaborativo de cada agente interviniente en el proceso. Por otro lado, el conocimiento en cada individuo es importante y más aún la gestión de este como un macroconocimiento institucional que permitirá que todos se desenvuelvan con seguridad y quienes estén integrando al equipo $\mathrm{y}$ esos puedan transmitirlo. Finalmente, ambas variables estudiadas se fusionan y complementan de manera singular para que generar mejoras a través de proyectos en los cuales se implementen herramientas que conlleven a una mejor práctica de su labor.

En este contexto la planificación estratégica va a significar cambio, y todo proceso de cambio no es sencillo de hacer ni de asumir. Implica tener conocimientos en distintas áreas para reflejar diferentes perspectivas para dar múltiples soluciones. Por otro lado, la capacitación debe ser permanente, de modo que, garantice no impartir información desfasada, además de utilizar instrumentos retrógrados, en lugar de las herramientas tecnológicas.

REFERENCIAS

Aranda, J y Salgado, E (2005). El diseño curricular y la planeación estratégica. Recuperado de https:// www.redalyc.org/pdf/1794/179421475003.pdf

Armijo, M. (2009). Planificación Estratégica e Indicadores de Desempeño en el Sector Público. Publicaciones. ILPES. Serie Manuales $N^{\circ} 69$. Santiago de Chile

Coronel, A. et all (2021). Planificación estratégica. Caso de estudio educativo Universidad Federico Villarreal. Recuperadode:http://www.scielo.org. $\mathrm{mx} /$ scielo.php?script=sci_arttext\&pid=S200778902021000200016\#B13Graw Hill, México.

Díaz, J. (2015). Re-significación y reconceptualización de la categoría calidad educativa: una nueva mirada, una nueva perspectiva. Revista Colombiana de Educación, Vol (68), pp. 173-194. Recuperado de: http://www.scielo.org.co/ scielo.php? script $=$ sci_abstract $\&$ pid $=$ S0120 39162015000100008\&lng=e 
García, J, et al. (2018). Internacionalización y planificación estratégica en apoyo a la calidad de la educación superior en México. Recuperado de: https://www.redalyc.org/ journal/4780/478055151004/html/

Minakata, A. (2000). El maestro que aprende: educación para una nueva época, Sinéctica (17), julio-diciembre. Recuperado de: http://www. scielo.org.mx/pdf/sine/n32/n32a8.pdf

Morales, A. (2011). Propuesta de un plan estratégico gerencial en la planificación educativa dirigida a los directores de la escuela básica. ARJÉ Revista de Postgrado FACE-UC. Vol. 5 No 9. JulioDiciembre 2011 / 35-63. Venezuela

Pérez-Soltero, A., et al (2013). Un diagnóstico de la gestión del conocimiento en las pymes del sector restaurantero para identificar áreas de mejora en sus procesos productivos. Omnia Science. IC, 2013 - 9(1): 153-183 - Online ISSN: 1697-9818 - Print ISSN: 2014-3214

Ramírez-Betancourt, F. D., Assafiri-Ojeda, E., Salgado-Cepero, G., \& Cruz-Hernández, L. (2020). Análisis de la eficacia de la gestión con enfoque externo en el sector privado. Ingeniería industrial, 41(3). http://scielo.sld.cu/scielo.php?pid=S1815$59362020000300007 \& \mathrm{script}=\mathrm{sci}$ arttext\&tlng=pt. [ Links ]

Rodríguez, J. (2018). Internacionalización y planificación estratégica en apoyo a la calidad de la educación superior en México. Recuperado de: https://www. redalyc.org/journal/4780/478055151004/ html/\#redalyc_478055151004_ref7 